\title{
Observation of the shadowing of cosmic rays by the Moon using a deep underground detector
}

M. Ambrosio, ${ }^{12}$ R. Antolini, ${ }^{7}$ C. Aramo,${ }^{7, l}$ G. Auriemma, ${ }^{14, a}$ A. Baldini, ${ }^{13}$ G. C. Barbarino, ${ }^{12}$ B. C. Barish, ${ }^{4}$ G. Battistoni,${ }^{6, b}$ R. Bellotti, ${ }^{1}$ C. Bemporad,${ }^{13}$ P. Bernardini,${ }^{10}$ H. Bilokon, ${ }^{6}$ V. Bisi,${ }^{16}$ C. Bloise,${ }^{6}$ C. Bower, ${ }^{8}$ S. Bussino ${ }^{14}$ F. Cafagna, ${ }^{1}$ M. Calicchio, ${ }^{1}$ D. Campana,${ }^{12}$ M. Carboni, ${ }^{6}$ M. Castellano, ${ }^{1}$ S. Cecchini ${ }^{2, c}$ F. Cei,${ }^{11,13}$ V. Chiarella, ${ }^{6}$ B. C. Choudhary, ${ }^{4}$ S. Coutu, ${ }^{11, \mathrm{~m}}$ L. De Benedictis, ${ }^{1}$ G. De Cataldo, ${ }^{1}$ H. Dekhissi, ${ }^{2,17}$ C. De Marzo, ${ }^{1}$ I. De Mitri, ${ }^{9}$ J. Derkaoui, ${ }^{2,17}$ M. De Vincenzi, ${ }^{14, \mathrm{e}}$ A. Di Credico, ${ }^{7}$ O. Erriquez, ${ }^{1}$ C. Favuzzi, ${ }^{1}$ C. Forti, ${ }^{6}$ P. Fusco, ${ }^{1}$ G. Giacomelli, ${ }^{2}$ G. Giannini, ${ }^{13, \mathrm{f}}$ N. Giglietto, ${ }^{1, *}$ M. Giorgini, ${ }^{2}$ M. Grassi, ${ }^{13}$ L. Gray,${ }^{4,7}$ A. Grillo, ${ }^{7}$ F. Guarino, ${ }^{12}$ P. Guarnaccia, ${ }^{1}$ C. Gustavino, ${ }^{7}$ A. Habig, ${ }^{3}$ K. Hanson,,${ }^{11}$ R. Heinz,${ }^{8}$ Y. Huang, ${ }^{4}$ E. Iarocci, ${ }^{6, g}$ E. Katsavounidis, ${ }^{4}$ E. Kearns,${ }^{3}$ H. Kim, ${ }^{4}$ S. Kyriazopoulou, ${ }^{4}$ E. Lamanna, ${ }^{14}$ C. Lane, ${ }^{5}$ D. S. Levin, ${ }^{11}$ P. Lipari, ${ }^{14}$ N. P. Longley, ${ }^{4, j}$ M. J. Longo, ${ }^{11}$ F. Maaroufi, ${ }^{2,17}$ G. Mancarella, ${ }^{10}$ G. Mandrioli, ${ }^{2}$ S. Manzoor, ${ }^{2, k}$ A. Margiotta Neri, ${ }^{2}$ A. Marini, ${ }^{6}$ D. Martello, ${ }^{10}$ A. Marzari-Chiesa, ${ }^{16}$ M. N. Mazziotta, ${ }^{1}$ C. Mazzotta, ${ }^{10}$ D. G. Michael, ${ }^{4}$ S. Mikheyev,${ }^{4,7, h}$ L. Miller, ${ }^{8}$ P. Monacelli, ${ }^{9}$ T. Montaruli, ${ }^{1}$ M. Monteno, ${ }^{16}$ S. Mufson, ${ }^{8}$ J. Musser, ${ }^{8}$ D. Nicoló, ${ }^{13, d}$ C. Orth,${ }^{3}$ G. Osteria, ${ }^{12}$ M. Ouchrif,${ }^{2,17}$ O. Palamara, ${ }^{10}$ V. Patera, ${ }^{6, g}$ L. Patrizii, ${ }^{2}$ R. Pazzi ${ }^{13}$ C. W. Peck, ${ }^{4}$ S. Petrera, ${ }^{9}$ P. Pistilli, ${ }^{14, \mathrm{e}}$ V. Popa,${ }^{2, \mathrm{i}}$ V. Pugliese,${ }^{14}$ A. Rainò, ${ }^{1}$ J. Reynoldson, ${ }^{7}$ F. Ronga, ${ }^{6}$ U. Rubizzo, ${ }^{12}$ C. Satriano, ${ }^{14, a}$ L. Satta, ${ }^{6, g}$ E. Scapparone, ${ }^{7}$ K. Scholberg, ${ }^{3}$ A. Sciubba, ${ }^{6, g}$ P. Serra-Lugaresi, ${ }^{2}$ M. Severi, ${ }^{14}$

M. Sioli, ${ }^{2}$ M. Sitta, ${ }^{16}$ P. Spinelli, ${ }^{1}$ M. Spinetti, ${ }^{6}$ M. Spurio, ${ }^{2}$ R. Steinberg, ${ }^{5}$ J. L. Stone,${ }^{3}$ L. R. Sulak, ${ }^{3}$ A. Surdo, ${ }^{10}$ G. Tarlè,${ }^{11}$ V. Togo, ${ }^{2}$ D. Ugolotti, ${ }^{2}$ M. Vakili, ${ }^{15}$ C. W. Walter, ${ }^{3}$ and R. Webb ${ }^{15}$

${ }^{1}$ Dipartimento di Fisica dell'Università di Bari and INFN, 70126 Bari, Italy

${ }^{2}$ Dipartimento di Fisica dell'Università di Bologna and INFN, 40126 Bologna, Italy

${ }^{3}$ Physics Department, Boston University, Boston, Massachusetts 02215

${ }^{4}$ California Institute of Technology, Pasadena, California 91125

${ }^{5}$ Department of Physics, Drexel University, Philadelphia, Pennsylvania 19104

${ }^{6}$ Laboratori Nazionali di Frascati dell'INFN, 00044 Frascati (Roma), Italy

${ }^{7}$ Laboratori Nazionali del Gran Sasso dell'INFN, 67010 Assergi (L'Aquila), Italy

${ }^{8}$ Department of Physics and Department of Astronomy, Indiana University, Bloomington, Indiana 47405

${ }^{9}$ Dipartimento di Fisica dell'Università dell'Aquila and INFN, 67100 L'Aquila, Italy

${ }^{10}$ Dipartimento di Fisica dell'Università di Lecce and INFN, 73100 Lecce, Italy

${ }^{11}$ Department of Physics, University of Michigan, Ann Arbor, Michigan 48109

${ }^{12}$ Dipartimento di Fisica dell'Università di Napoli and INFN, 80125 Napoli, Italy

${ }^{13}$ Dipartimento di Fisica dell'Università di Pisa and INFN, 56010 Pisa, Italy

${ }^{14}$ Dipartimento di Fisica dell'Università di Roma "La Sapienza" and INFN, 00185 Roma, Italy

${ }^{15}$ Physics Department, Texas A\&M University, College Station, Texas 77843

${ }^{16}$ Dipartimento di Fisica Sperimentale dell'Università di Torino and INFN, 10125 Torino, Italy

${ }^{17}$ Also at Faculty of Sciences, University Mohamed I, B.P. 424 Oujda, Morocco

(MACRO Collaboration)

(Received 11 June 1998; published 20 November 1998)

\begin{abstract}
Using data collected by the MACRO experiment during the years 1989-1996, we show evidence for the shadow of the Moon in the underground cosmic ray flux with a significance of $3.6 \sigma$. This detection of the shadowing effect is the first by an underground detector. A maximum-likelihood analysis is used to determine that the angular resolution of the apparatus is $0.9^{\circ} \pm 0.3^{\circ}$. These results demonstrate MACRO's capabilities as a muon telescope by confirming its absolute pointing ability and quantifying its angular resolution.

[S0556-2821(98)04723-7]

PACS number(s): 96.40.Cd, 13.85.Tp, 96.40.Tv
\end{abstract}

\footnotetext{
*Corresponding author.

${ }^{a} A$ lso at Università della Basilicata, 85100 Potenza, Italy.

${ }^{\mathrm{b}}$ Also at INFN Milano, 20133 Milano, Italy.

${ }^{\mathrm{c}}$ Also at Istituto TESRE/CNR, 40129 Bologna, Italy.

${ }^{\mathrm{d}}$ Also at Scuola Normale Superiore di Pisa, 56010 Pisa, Italy.

eAlso at Dipartimento di Fisica, Università di Roma Tre, Roma, Italy.

${ }^{\mathrm{f}}$ Also at Università di Trieste and INFN, 34100 Trieste, Italy.

${ }^{\mathrm{g}}$ Also at Dipartimento di Energetica, Università di Roma, 00185 Roma, Italy.

${ }^{\mathrm{h}}$ Also at Institute for Nuclear Research, Russian Academy of Science, 117312 Moscow, Russia.

${ }^{\mathrm{i}}$ Also at Institute for Space Sciences, 76900 Bucharest, Romania.

${ }^{j}$ Also at Swarthmore College, Swarthmore, PA 19081.

${ }^{\mathrm{k}}$ Also at RPD, PINSTECH, P.O. Nilore, Islamabad, Pakistan.

${ }^{1}$ Also at INFN Catania, 95129 Catania, Italy.

mAlso at Department of Physics, Pennsylvania State University, University Park, PA 16801.
} 


\section{INTRODUCTION}

MACRO is a large area underground detector located in Hall B of the Gran Sasso National Laboratory (LNGS) in Italy at an average depth of 3700 meter of water equivalent (mwe). The full apparatus has dimensions $76.5 \mathrm{~m} \times 12 \mathrm{~m}$ $\times 9.6 \mathrm{~m}$. The detector's active technologies include liquid scintillation counters and streamer tubes. Low-radiation crushed rock separates the active detector planes. Normally incident muons from above lose $\approx 1.5 \mathrm{GeV}$ in traversing the detector; at the surface, thoroughgoing muons have energies in excess of $\sim 3 \mathrm{TeV}$, corresponding to primary cosmic rays of energy $>7 \mathrm{TeV}$. The average energy of the muons detected by MACRO is $\sim 300 \mathrm{GeV}$. A detailed description of the detector can be found in [1].

MACRO was primarily designed to search for monopoles and rare particles in cosmic rays, including high energy neutrinos and muons from cosmic point sources [1]. Since high energy neutrinos are identified as upward-going muon secondaries from neutrino interactions in the rock below the detector, MACRO functions as a muon telescope [2] in its search for muons and neutrinos from cosmic sources. The purpose of this investigation is to verify its absolute pointing and quantify its angular resolution to point sources. Confidence in pointing is clearly essential for a telescope, and a preliminary determination of MACRO's pointing has been made by bootstrapping highway surveys from the surface into the tunnel. However, a check of the accuracy of this determination is important. Further, reconstructed muon tracks point back to an area on the sky whose width depends on the intrinsic angular resolution of the detector as well as multiple Coulomb scattering in the rock overburden. Consequently, the quality of MACRO as a muon telescope depends on its angular resolution. As the required search region around a source shrinks with improving resolution, the contribution of events from the flat or slowly varying background also decreases relative to the signal.

Traditional astronomical telescopes use observable point sources to determine pointing and angular resolution. Despite early reports of their existence [3], however, there are as yet no established cosmic muon sources. In the absence of cosmic sources, we adopt the approach of the CYGNUS [4], CASA [5], and Tibet [6] air shower arrays who used the Moon as a fiducial object. By blocking cosmic ray primaries, the Moon appears as a cosmic ray antisource or "shadow" [7] which can be used to verify MACRO's absolute pointing and to determine its angular resolution.

\section{MUON DATA SAMPLE AND THE EXPECTED BACKGROUND}

\section{A. Muon data sample}

MACRO consists of six nearly identical units called supermodules, each of dimension $12.6 \mathrm{~m} \times 12 \mathrm{~m} \times 9.6 \mathrm{~m}$. The lower $4.8 \mathrm{~m}$ of each supermodule consists of 10 horizontal planes of streamer tubes of dimension $12 \mathrm{~m} \times 6 \mathrm{~m}$. The 8 innermost planes are separated by 7 layers of $60 \mathrm{gm} / \mathrm{cm}^{2}$ absorber. The two outermost planes are each separated from the next nearest streamer tube plane by a $25 \mathrm{~cm}$ layer of liquid scintillator. The lateral walls consist of stacked tanks of liquid scintillator, $25 \mathrm{~cm}$ thick, sandwiched between six vertical streamer tubes planes. The upper $4.8 \mathrm{~m}$ of each supermodule (the attico) is a hollowed out version of the lower supermodule. It consists of a top plane of liquid scintillator with two streamer tube planes both above and below, and lateral walls of liquid scintillator with three vertical streamer tubes planes outside and inside. All streamer tube wires are read out, providing the $\mathrm{X}$ coordinate on the horizontal planes and the $\mathrm{Z}$ coordinate on the vertical planes. On the horizontal planes the second coordinate, $\mathrm{D}$, is obtained by horizontal aluminum strips oriented $26.5^{\circ}$ with respect to the streamer tube axis. These strips allow stereoscopic reconstruction. For a complete description of the detector, see [1].

The muon sample used for the present analysis includes all events collected from the start of MACRO data taking in February 1989 through the end of 1996 . The sample totals $39.3 \times 10^{6}$ events collected over $2.7 \times 10^{3}$ live days. During the first part of this period the apparatus was under construction. Long running periods included one supermodule without the attico $\left(A_{e f f} \Omega \approx 1010 \mathrm{~m}^{2} \mathrm{sr}\right)$, six supermodules without attico $\left(A_{e f f} \Omega \approx 5600 \mathrm{~m}^{2} \mathrm{sr}\right)$ and finally the full six supermodules with attico $\left(A_{e f f} \Omega \approx 6600 \mathrm{~m}^{2} \mathrm{sr}\right)$. Approximately $60 \%$ of the data sample was obtained during periods when MACRO had full acceptance.

The criteria used to select events for this analysis were designed to optimize the quality of reconstructed tracks. The selected events are consequently those which most accurately point back to their origin on the celestial sphere. The specific data cuts used in this analysis are listed below.

Run cuts

(1) Runs less than $1 \mathrm{~h}$ in length were cut since short runs were usually abnormally terminated and often contained malfunctioning hardware or software.

(2) Runs with large numbers of UT clock errors were cut.

Event cuts

(1) Only single and double muon events were retained; events with multiplicity $>2$ were cut. The more complicated task of reconstructing multimuon tracks is more likely to introduce tracking errors.

(2) Successful track reconstruction requires a minimum of four crossed horizontal streamer tube planes; events with fewer than four crossed horizontal planes were cut.

(3) Events crossing fewer than 3 streamer tube planes in the lower supermodules were cut. This cut removes low energy events that pass through the attico without crossing any rock absorber layers. Such events have large multiple Coulomb scattering angles and so are spread widely with respect to their point of origin on the celestial sphere.

(4) Reconstructed tracks with $\chi^{2} / N_{\mathrm{DF}}>1.5$ were cut. This cut removes events with poorly reconstructed tracks as well as events with large numbers of hits outside the track.

(5) Events with different reconstructed multiplicity in the two streamer tube views were cut.

(6) Events with UTC clock errors were cut since the correct time is necessary to project the track back onto the celestial sphere.

These selection criteria reduce the sample size to 30.51 $\times 10^{6}$ muons. 
The topocentric position of the Moon was computed at the arrival time of each event in the sample using the database of ephemerides available from the Jet Propulsion Laboratory, JPLEPH [8]. A correction for the parallax due to MACRO's instantaneous position on Earth was applied to each ephemeris position [9]. Since the parallax correction requires an accurate computation of the Earth-Moon distance, this calculation also results in an accurate determination of angular size subtended by the Moon at MACRO.

The muon events in a window $10^{\circ}$ on a side and centered on the Moon were selected for further analysis. There are $2.3 \times 10^{5}$ events that pass all cuts in this window.

\section{B. Expected background}

Twenty-five background samples were generated for each run used in the analysis. These backgrounds were constructed by coupling the direction of each muon in the run with the times of 25 randomly selected muons from the same run [2]. The 25 background samples were then processed using the same procedure as the muon data sample.

\section{SHADOW OF THE MOON}

\section{A. Event deficit around the Moon}

Each muon event in the window was sorted into bins of equal angular width as a function of angular distance from the Moon center. The angular width of each bin was $0.125^{\circ}$, which gives a solid angle for the $i_{t h}$ bin of $\Delta \Omega_{i}=(2 i-1)$ $\times 0.05 \mathrm{deg}^{2}$. Once filled, the contents of each bin, $N_{i}$, were divided by the solid area of the bin, resulting in the distribution, $\Delta N / \Delta \Omega$ which approximates the differential event density as a function of angular distance from the Moon center. Figure 1 shows as data points the computed differential event density. The errors on the data points are statistical. Superposed on this distribution is the expected event density in the absence of the shadowing effect, $\Delta N^{b k d} / \Delta \Omega$. This background distribution was determined by averaging over the 25 background samples the number of events in each solid angle bin, and then dividing the result by the solid angle of the bin. This nearly flat distribution is described reasonably well by a constant event density $\approx 735 \mathrm{deg}^{-2}$. The shadowing effect, or the deviation of the angular event density from the background, is clearly evident. This figure confirms that reconstructed muon tracks can be accurately pointed back to celestial coordinates, thus confirming MACRO's absolute pointing ability.

We have made a simple estimate of the significance of this detection of the Moon shadow using the information given in Fig. 1. First, we computed the integral event deficit as a function of the angular distance from the Moon center. Out to bin $n$, the integral event deficit, $\Delta_{n} N^{d e f}$, is given by

$$
\Delta_{n} N^{d e f}=\sum_{i=1}^{n}\left[N_{i}^{b k d}-N_{i}\right] .
$$

This distribution represents the cumulative number of events that the data distribution differs from the flat background and is shown in Fig. 2. This figure shows that the deficit in- creases until the shadow can no longer be distinguished from the background at $\sim 0.9^{\circ}$. Out to $0.9^{\circ}$ the integral deficit can be approximated by $\Delta N^{d e f} \approx 165 \alpha$, where $\alpha$ is the angular distance from the Moon center; this simple approximation is shown as a dashed line in Fig. 2. An estimate of the significance is then given by

$$
\frac{\Delta N^{d e f}}{\sqrt{N^{b k d}}}=\frac{165 \alpha}{\sqrt{735 \pi \alpha^{2}}} \approx 3.5 .
$$

This computation shows that the Moon shadow detection has a significance of approximately $3.5 \sigma$.

\section{B. Maximum likelihood analysis}

In the simple deficit analysis above, we have implicitly assumed that the position of the Moon's shadow is known and we have binned the events using this information. We now relax that assumption and search for the Moon shadow in a direction-independent way with the maximum likelihood method of COS-B [10], a technique first described in detail by Cash [11]. This method is based on a priori knowledge of the MACRO point spread function (MPSF) detector.

We have determined the MPSF using the observed space angle distribution of double muons. As shown in [12], the number of double muons as a function of lateral separation is a power law distribution that falls with a scale length of $\sim 15 \mathrm{~m}$. Muon pairs produced in a primary interaction at 20 $\mathrm{km}$ therefore have typical initial separation angles $\leqslant .05^{\circ}$. Since "double" muons initially move along virtually parallel paths, the distribution of their separation angles is a good measure of the deviations introduced into their tracking parameters by both scattering in the mountain overburden and the detector's intrinsic angular resolution. The space angle distribution of "double" muons must be divided by $\sqrt{2}$ to obtain the MPSF since both muons deviate from their initial trajectories. In Fig. 3 the MPSF, as determined from 1044877 muon pairs, is shown in altitude and azimuthal coordinates. Figure 3 shows the strongly peaked, nonGaussian behavior of the MPSF.

To find the most likely position of the Moon, we compare the two dimensional distribution of muons in the window centered on the Moon with the expected background events in the same window. In this analysis, each muon event is first sorted into a grid of equal solid angle bins $\left(\Delta \Omega=0.125^{\circ}\right.$ $\left.\times 0.125^{\circ}=1.6 \times 10^{-2} \mathrm{deg}^{2}\right)$. The shadowing source of strength $S_{M}$ at fixed position $\left(x_{s}, y_{s}\right)$ that best fits the data is then found by minimizing

$$
\chi^{2}\left(x_{s}, y_{s}, S_{M}\right)=2 \sum_{i=1}^{n_{b i n}}\left[N_{i}^{e x}-N_{i}+N_{i} \ln \frac{N_{i}}{N_{i}^{e x}}\right]
$$

where the sum is over all bins in the window [13]. Here $N_{i}$ is the number of events observed in each bin $i, N_{i}^{e x}$ is the expected number of events in bin $i$, and $n_{b i n}$ is the number of bins in the grid. This expression assumes that a Poissonian process is responsible for the events seen in each bin. The expected number of events in bin $i$ is given by 


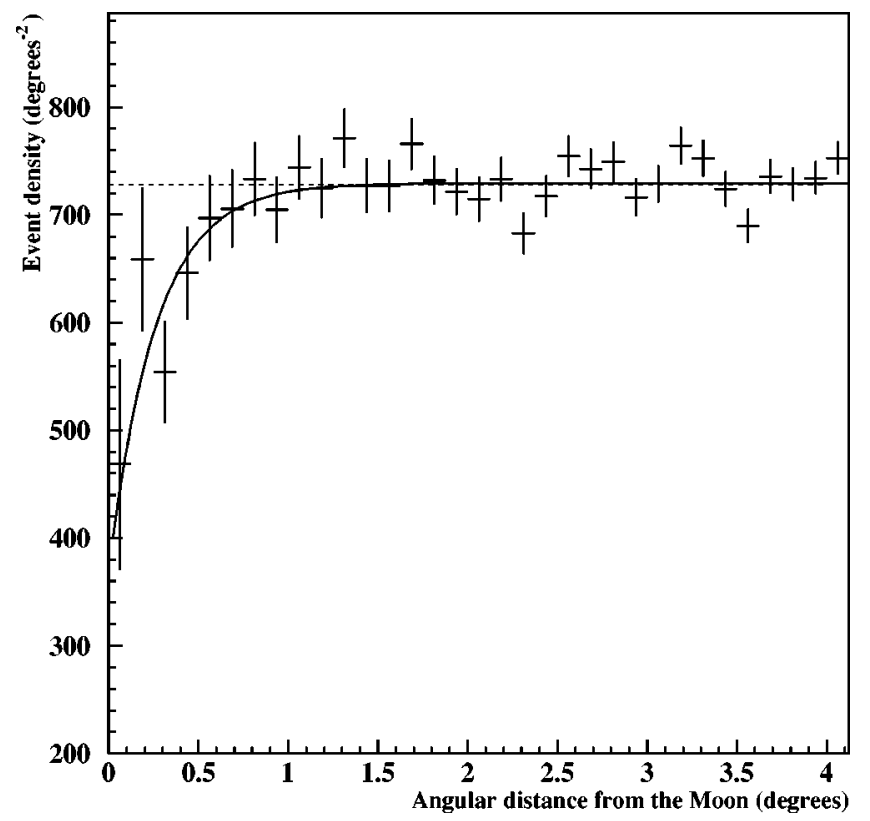

FIG. 1. The event density vs angular distance from the Moon center in bins of equal angular width. The width of each bin is $0.125^{\circ}$. The dashed curve is the average expected background computed from 25 background samples. The solid curve shows the expected event density as computed for an angular resolution of the MACRO apparatus of $0.90^{\circ}$.

$$
N_{i}^{e x}=N_{i}^{b k d}-S_{M} \cdot \mathcal{P}\left(x_{s}-x_{i}, y_{s}-y_{i}\right)
$$

where $N_{i}^{b k d}$ is the average number of background events at position $\left(x_{i}, y_{i}\right)$, and $S_{M} \cdot \mathcal{P}\left(x_{s}-x_{i}, y_{s}-y_{i}\right)$ is the number of events removed from bin $i$ by the shadow of the Moon. Here

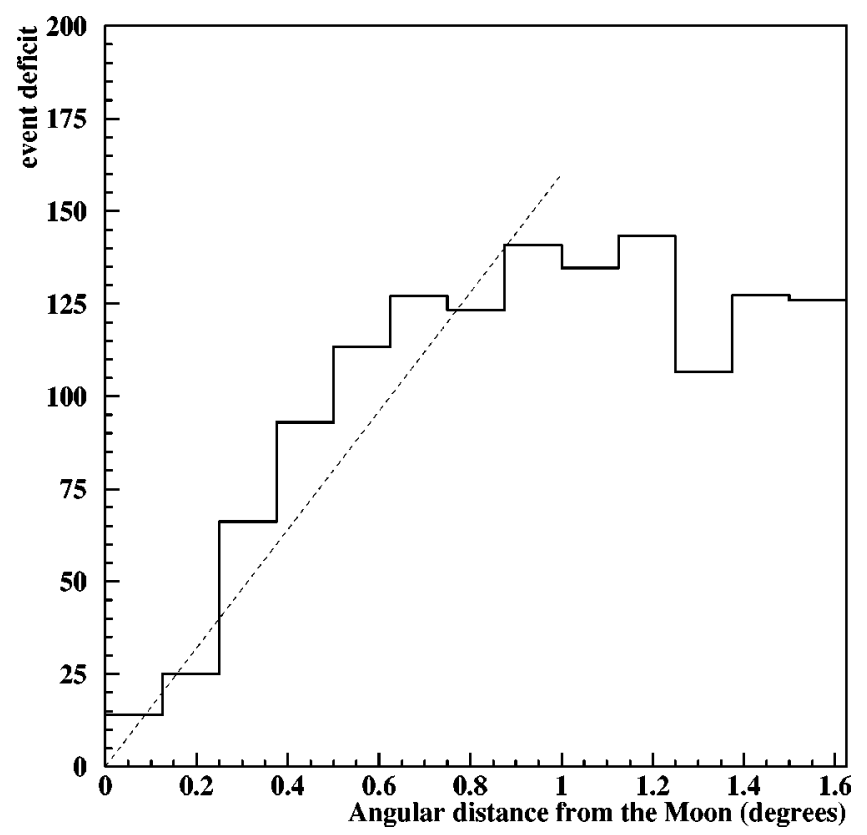

FIG. 2. Integral event deficit vs angular distance from the Moon center. Superposed on the integral event deficit is an approximate fit to the distribution out to $0.9^{\circ}: \Delta N^{d e f}=165 \alpha$, where $\alpha$ is the angular distance from the Moon center.
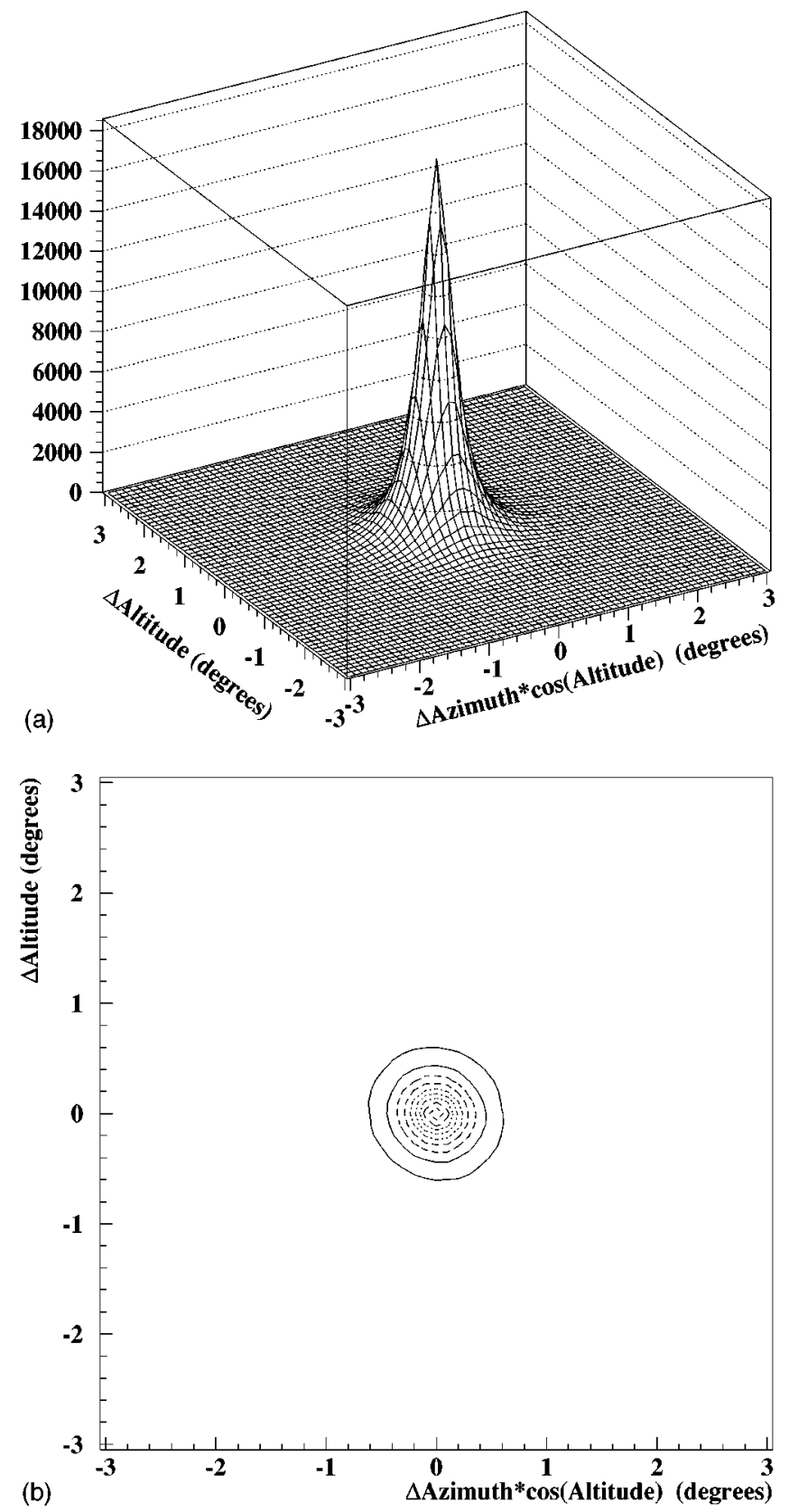

FIG. 3. (a) The point spread function of the MACRO apparatus (MPSF) derived from the space angle distribution of 1044877 double muons. (b) Contour diagram of the MPSF. The contours levels are equally spaced between 2000 and 18000 muons. The MPSF is circularly symmetric in this coordinate system.

$\mathcal{P}\left(x_{s}-x_{i}, y_{s}-y_{i}\right)$ is the MPSF, modified for the finite size of the Moon's disk, computed at the point $\left(x_{i}, y_{i}\right)$ when the shadowing source is at $\left(x_{s}, y_{s}\right)$. The MPSF was modified by first selecting random positions on a disk with the average lunar radius, $0.26^{\circ}$, and then drawing offsets from these positions from the MPSF distribution These new positions were rebinned into a new histogram and the resulting distribution normalized to unit area. The modified point spread function is shown in Fig. 4. In this figure, the unmodified, normalized MPSF is shown in Fig. 4(a); the MPSF modified for the Moon's finite disk is shown in Fig. 4(b). In Figs. 4(c) and 


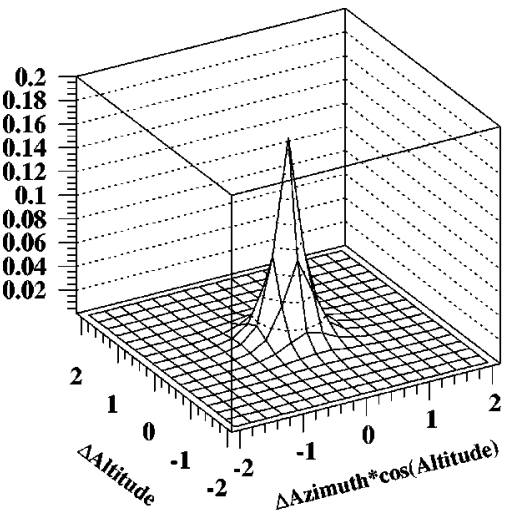

(a)

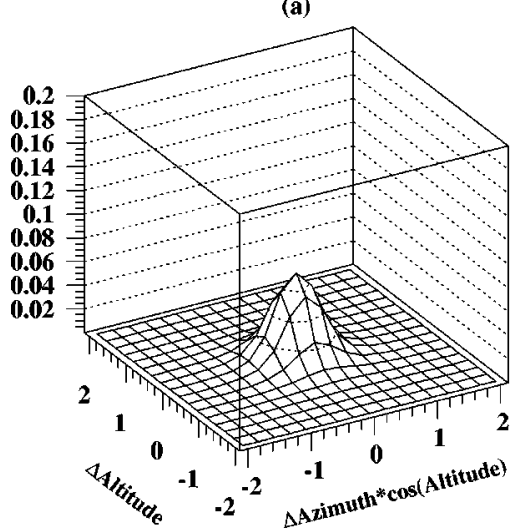

(c)

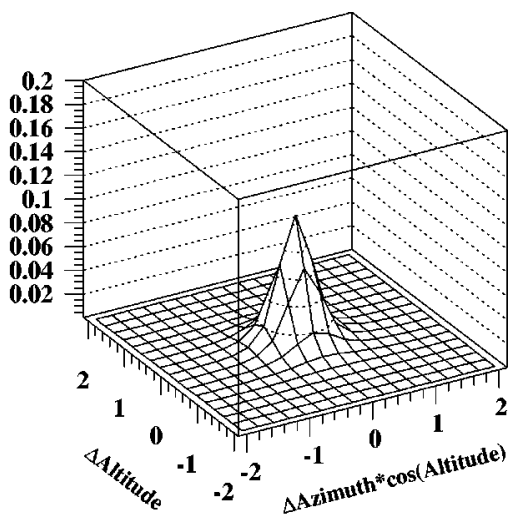

(b)

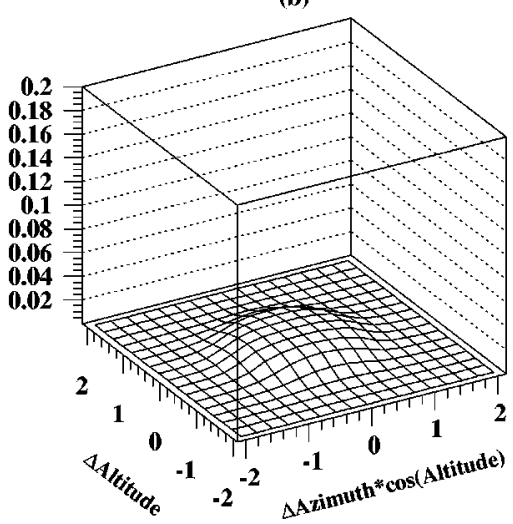

(d)

FIG. 4. (a) The MPSF, normalized to unit area from Fig. 3(a). (b) The MPSF modified by a disk of radius $0.26^{\circ}$, the average lunar radius, and then normalized to unit area; this is the modified MPSF used in the likelihood analysis. (c) The MPSF modified by a disk of radius $0.4^{\circ}$ and then normalized. (d) The MPSF modified by a disk of radius $1.0^{\circ}$ and then normalized.

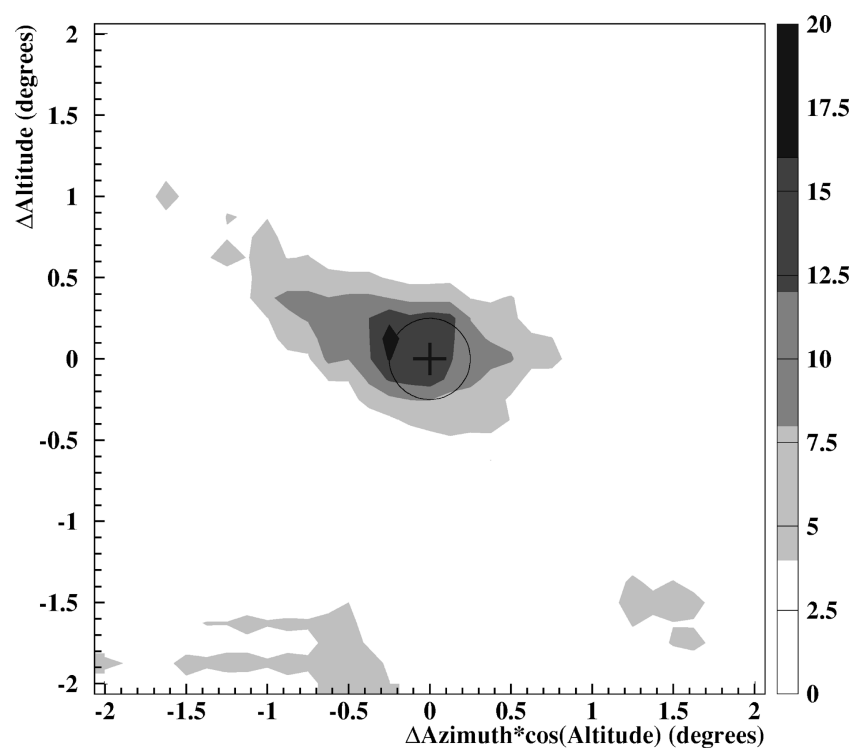

FIG. 5. The two dimensional distribution of $\lambda$ in bins of equal solid angle in the Moon window The axes are offsets from the Moon center. The fiducial position of the Moon, at position $(0,0)$, is marked by a + ; a circle corresponding to the average lunar radius, $0.26^{\circ}$, is centered at this position. The $\lambda$ grey scale is given at the right margin of the figure. The maximum of this distribution, $\Lambda=18.3$, is offset from the fiducial Moon position at $\Delta$ azimuth $=-0.25^{\circ}$ and $\Delta$ altitude $=+0.125^{\circ}$. 4(d) the MPSF has been modified by still larger shadowing disks. The effect of the finite size of the Moon's disk does not have a large impact on the analysis.

Finally, the shadow strength $S_{M}$ that minimizes $\chi^{2}$ was computed for every grid point in the window. This minimum $\chi^{2}\left(x_{s}, y_{s}, S_{M}\right)$ was then compared with $\chi^{2}(0)$ for the null hypothesis that no shadowing source is present in the window $\left(S_{M}=0\right)$ :

$$
\lambda=\chi^{2}(0)-\chi^{2}\left(x_{s}, y_{s}, S_{M}\right) .
$$

The most likely position of the Moon is the bin in which the maximum $\lambda, \lambda^{\max } \equiv \Lambda$, is found. Since there is only one free parameter, $S_{M}, \lambda$ behaves like $\chi_{1}^{2}$, a $\chi^{2}$ distribution with one degree of freedom [10]. The significance of the Moon detection is given by $\chi_{1}^{2}(\Lambda)$.

In Fig. 5 we show the results of this analysis in a window $4.375^{\circ} \times 4.375^{\circ}$ centered on the Moon. This window has been divided into $35 \times 35$ cells, each having dimensions $0.125^{\circ}$ $\times 0.125^{\circ}$. In this figure, $\lambda$ is displayed in gray scale format for every bin in the Moon window. Also shown is the fiducial position of the Moon and a circle centered at this position corresponding to the average lunar radius, $0.26^{\circ}$. The maximum $\Lambda=18.3$ is found somewhat offset from the fiducial Moon position at $\Delta$ azimuth $=-0.25^{\circ}, \quad \Delta$ altitude $=+0.125^{\circ}$ and provides further confirmation of MACRO's absolute pointing. The value of the shadow strength at this 


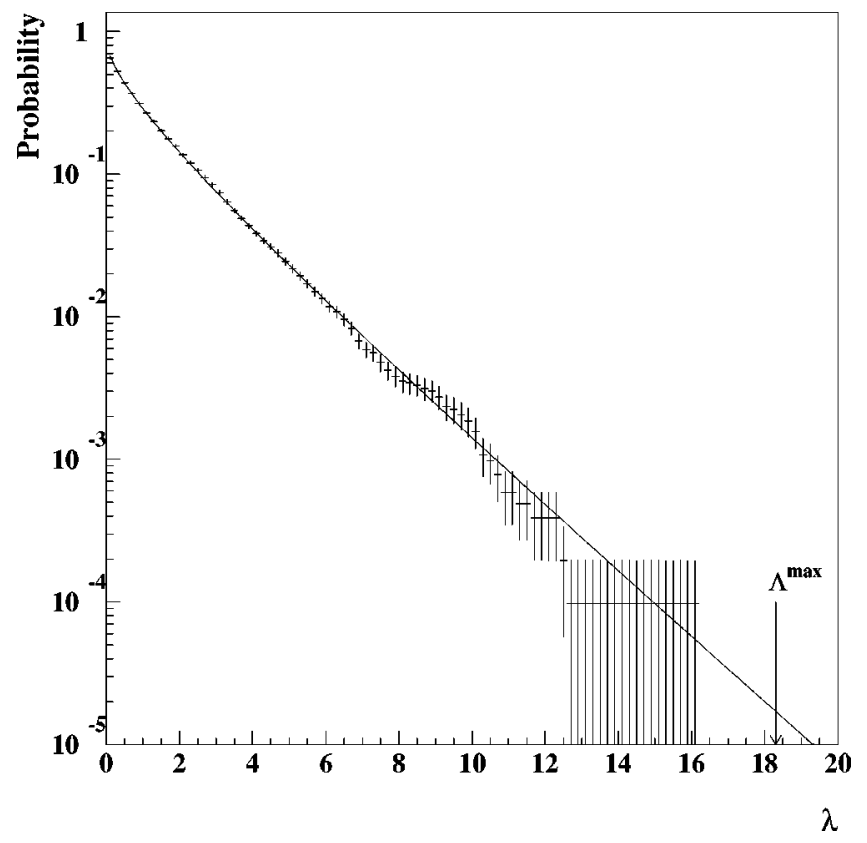

FIG. 6. The cumulative distribution of 10224 values of $\lambda$ for all bins in the 71 off-source windows. Superposed on this distribution, as a solid curve, is the cumulative $\chi_{1}^{2}$ distribution. The arrow marks $\Lambda=18.3$ from the likelihood analysis.

position, $S_{M}=153.9 \pm 37$ events, agrees well with the observed value 155 events. Although the displacement from the fiducial position of the Moon is not of high significance, we note that it is consistent with the deflection of cosmic ray primaries by the geomagnetic field [6].

We have verified the properties of the $\lambda$ distribution by constructing 71 other windows similar to the Moon window,

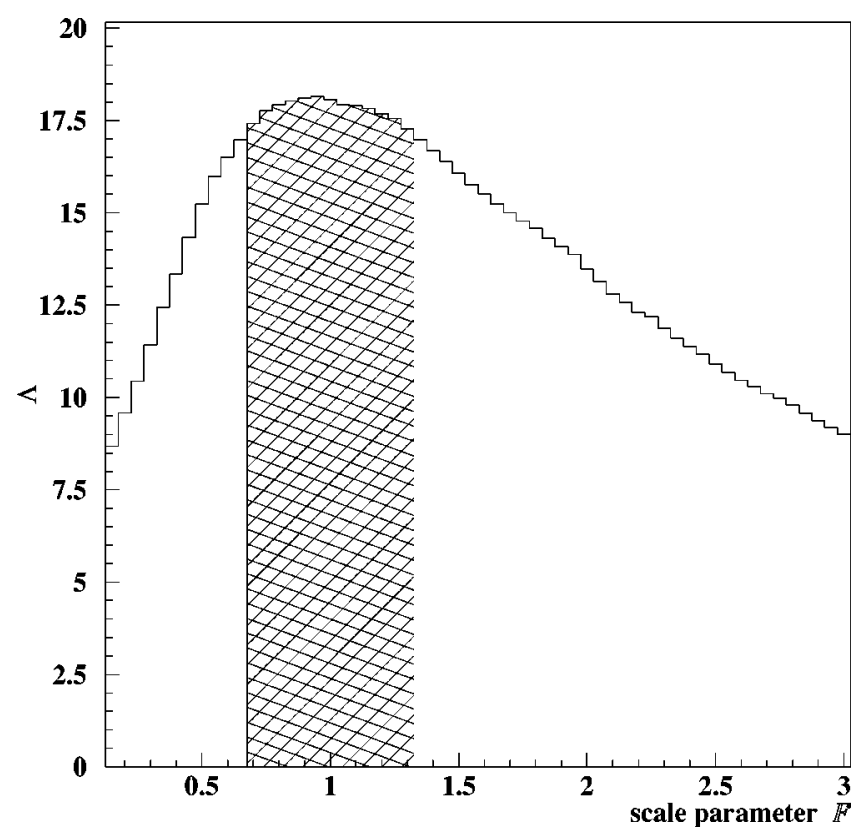

FIG. 7. The distribution of $\Lambda$ as a function of $\mathcal{F}$. The maximum $\Lambda, \Lambda^{*}$, is found for $\mathcal{F}=1.0$. The shaded area shows the region where $\Lambda^{*}$ falls by 1.0 , or the $1 \sigma$ confidence interval for $\mathcal{F}$ [3].
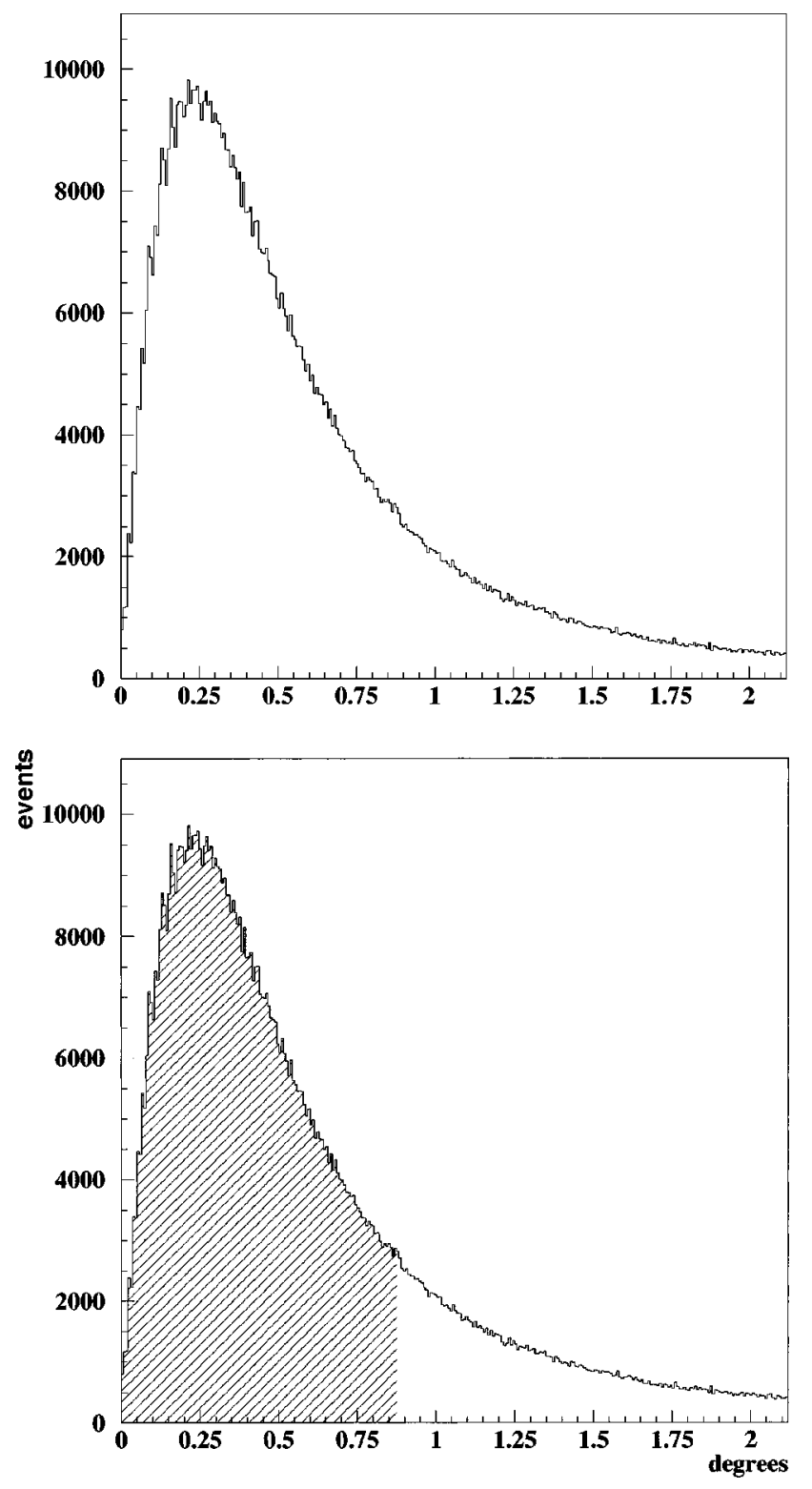

FIG. 8. The space angle distribution of double muons. The angular resolution of the MACRO apparatus, defined as the cone angle containing $68 \%$ of the events from a point source, is shown as a shaded region. With this definition, MACRO's angular resolution is $0.90^{\circ}$.

each displaced from the next by $5^{\circ}$ in right ascension. For each off-source window, we followed the procedure used for the Moon window in computing the expected background. To avoid edge effects associated with a source near the edge of a window, we only evaluated $\lambda$ for the central $12 \times 12$ bins. In Fig. 6 we have plotted the cumulative distribution of the $12 \times 12 \times 71=10224$ values of $\lambda$ for the 71 off-source windows. Superposed on this distribution as a solid curve is the cumulative $\chi_{1}^{2}$ distribution. The distributions agree well (the structure seen at $7 \leqslant \lambda \leqslant 10$ is due to round-off error). Thus, the probability of the detection of the shadow of the Moon at this bin location is $p\left(\chi_{1}^{2}=18.3\right) \leqslant 1.9 \times 10^{-5}$. However, we would have considered the detection of the Moon shadow equally secure had the maximum been found at any 
of the 9 bins within the geometric shadow, which reduces the probability of detection to $p \leqslant 1.7 \times 10^{-4}$. The significance of the detection is therefore $3.6 \sigma$ and the results of the likelihood analysis and the deficit analysis are in excellent agreement.

As a cross-check, a similar analysis in right ascension and declination was performed. The results were equivalent, as required.

\section{ANGULAR RESOLUTION OF THE MACRO APPARATUS}

As shown in Fig. 3, the MPSF cannot be fit by a simple Gaussian function. Thus, the technique used by the CYGNUS [4], CASA [5], and Tibet [6] air shower arrays, where such a simplification was possible, must be modified. The air shower experiments find their PSF by rigidly scaling the dispersion of their Gaussian resolution functions and then computing the likelihood function for the detection of the Moon shadow for each scaled value of $\sigma$. The maximum likelihood defines the $\sigma$ to be used in the computation of the angular resolution. In our approach, we first defined a scale parameter $\mathcal{F}$ that rigidly scales the modified MPSF by the factor $\mathcal{F}$ :

$$
\widetilde{\mathcal{P}}\left(x_{s}-x_{i}, y_{s}-y_{i} ; \mathcal{F}\right) .
$$

We then repeated the likelihood analysis in the Moon window for different values of $\mathcal{F}$. We assume that the value of $\mathcal{F}$ that maximizes $\Lambda$ gives the best $\widetilde{\mathcal{P}}$ for computing the angular resolution.

The distribution of $\Lambda$ as a function of $\mathcal{F}$ is shown in Fig. 7. The maximum $\Lambda, \Lambda^{*}$ is found for $\mathcal{F}=1.0$ which implies that the unscaled space angle distribution of double muons should be used to determine the angular resolution of the MACRO apparatus. Many definitions have been used for the angular resolution $[4,5,6]$. We choose the cone of angle $\theta_{68 \%}$ that contains the $68 \%$ of the events from a point-like source.
In Fig. 8 we show the space angle distribution of double muons. Using our definition of the angular resolution, Fig. 8 gives $\theta_{68 \%}=0.90^{\circ}$. The $1 \sigma$ error limits on the angular resolution can be estimated from the interval of $\mathcal{F}$ in which $\Lambda^{*}$ falls by 1.0 [13]. The shaded region in Fig. 7 shows this interval to be $\mathcal{F}=1.0 \pm 0.35$. Using these values to scale the double muon distribution, we find that the angular resolution is $\theta_{68 \%}=0.9^{\circ} \pm 0.3^{\circ}$.

In Fig. 1 we show as a solid line the expected event density in the Moon window for an angular resolution of the MACRO apparatus of $0.90^{\circ}$. The model used in this computation is given by

$$
\frac{\Delta N}{\Delta \Omega}=\Delta\left[N^{b k d}-S_{M} \cdot \widetilde{\mathcal{P}}(\alpha ; \mathcal{F}=1.0)\right] / \Delta \Omega,
$$

where $\alpha=\sqrt{\left(x_{s}-x_{i}\right)^{2}+\left(y_{s}-y_{i}\right)^{2}} \mathrm{DF}$ is the angular distance from the Moon center. The model fits the data well $\left[\chi^{2} / N_{\mathrm{DF}}=(48.7 / 47)\right]$.

\section{CONCLUSIONS}

The MACRO detector at a mean depth of 3700 mwe, operational since February 1989, has collected a muon sample of about $39 \times 10^{6}$ events. Using this sample we have searched for the Moon shadow cast in the cosmic ray sky at primary energies $\sim 10-15 \mathrm{TeV}$. In the deficit analysis, we find an event deficit around the Moon of significance $3.5 \sigma$. With a maximum likelihood analysis, we confirm the detection of the Moon's shadow with significance of 3.6\%. This is the first detection of the Moon shadow underground. Our estimate of the angular resolution is $\theta_{68 \%}=0.9^{\circ} \pm 0.3^{\circ}$.

These results demonstrate MACRO's capabilities as a muon telescope by confirming its absolute pointing ability and quantifying its angular resolution. This investigation shows that the MACRO detector has the capability of detecting signals from cosmic sources by observing the secondary cosmic muon underground.
[1] S. Ahlen et al., Nucl. Instrum. Methods Phys. Res. A 324, 337 (1993); M. Ambrosio et al. (in preparation).

[2] S. Ahlen et al., Astrophys. J. 412, 301 (1993).

[3] M. L. Marshak et al., Phys. Rev. Lett. 54, 2079 (1985); M. L. Marshak et al., ibid. 55, 1965 (1985).

[4] D. E. Alexandreas et al., Phys. Rev. D 43, 1735 (1991).

[5] A. Borione et al., Phys. Rev. D 49, 1171 (1994).

[6] M. Amenomori et al., Phys. Rev. D 47, 2675 (1993).

[7] G. W. Clark, Phys. Rev. 108, 450 (1957).

[8] E. M. Standish, X. X. Newhall, J. G. Williams, and W. F.
Folkner, "JPL Planetary and Lunar Ephemerides,' JPL IOM 314, 10 (1995).

[9] P. Duffett-Smith, Practical Astronomy with Your Calculator, 3rd ed. (Cambridge University Press, Cambridge, England, 1988), p. 150.

[10] A. M. T. Pollock et al., Astron. Astrophys. 94, 116 (1981).

[11] W. Cash, Astrophys. J. 228, 939 (1979).

[12] M. Ambrosio et al., Phys. Rev. D 56, 1407 (1997).

[13] Particle Data Group, R. M. Barnett et al., Phys. Rev. D 54, 1 (1996), p. 160. 